\title{
Female sterilisations with Filshie clips: What is the risk failure? A retrospective survey of 30000 applications
}

\author{
Gabor T Kovacs, MD, FRACOG, Professor; Anthony J Krins, FRACOG, MRCOG, Senior Lecturer, Monash Medical School, Box \\ Hill Hospital, Victoria, Australia
}

Correspondence: Professor Gabor T Kovacs, Division of Obstetrics \& Gynaecology, Box Hill Hospital, Nelson Road, Box Hill, Victoria 3128, Australia.Tel: (61) 39895 3379. Fax: (61) 39895 3143.Email: gab.kovacs@ boxhill.org.au

(Accepted $4^{\text {th }}$ August 2001)

The Journal of Family Planning and Reproductive Health Care 2002: 28(1): 34-35

\begin{abstract}
Objective. To assess the failure rate of Filshie clip sterilisations in Victoria, Australia.

Design. Retrospective study between 1994 and 1998.

Setting. All specialist gynaecologists practising in the state of Victoria, Australia.

Interventions. Questionnaires (up to three) followed by telephone calls if necessary.

Main outcome measures. A response rate of 276/277 (99.6\%) was obtained. All pregnancies were recorded.

Results. Seventy-three failures were notified from an estimated 30000 Filshie clip sterilisations carried out in the study period.

Conclusions. The estimated failure rate of Filshie clip sterilisations in the state of Victoria carried out by specialist gynaecologists was between 2 and 3 per 1000 sterilisation operations.
\end{abstract}

\section{Introduction}

Following a review of female sterilisation, ${ }^{1}$ the Family Planning Federation of Australia recommended that 'the preferred method of tubal occlusion is to apply Filshie clips at the mid isthmus'. ${ }^{2}$ Consequently, a failure rate of 3 per 1000 sterilisation operations was the figure given to women planning sterilisation. Recently it has been suggested that the 10-year cumulative failure rate of female sterilisation is in the range of 7.5 to 36.5 per 1000 sterilisation operations. ${ }^{3}$ Although Filshie clips were not included in this study as they are not available in the USA, their efficacy has only had limited evaluation. A study in South Africa of 789 postpartum Filshie clip sterilisations showed a failure rate of $1.01 \%$ over 2 years. ${ }^{4}$ A New Zealand study which included Filshie clips for sterilisations performed in 1988 and 1989 showed a failure rate of $1.2 \% .^{5}$ A Danish study of Filshie clips showed a failure rate of $1.36 \% .^{6}$ A series of 425 Filshie clip sterilisations carried out by the inventor of the clip (Marcus Filshie) recruited 425 patients with only one reported failure due to operator error. ${ }^{7}$

Recently the results of a prospective study of Filshie clip sterilisations co-ordinated by Family Health International were reported. This was a prospective study of seven centres from five countries in which 916 women were sterilised by means of laparoscopically applied Filshie clips between 1984 and 1990. The life table pregnancy rate at 12 months' follow-up was 1.2 per 1000 sterilisation operations. 8

Recently there have been reports of Filshie clip failures attributed to the miscalibration of applicators. Following warnings from the Australian distributors of the Filshie clip and the Royal Australian and New Zealand College of Obstetrics and Gynaecologists (RANZCOG), there is confusion amongst health professionals about how effective Filshie clip female sterilisation is. It was therefore decided to carry out a quality assurance project under the auspices of the Victorian State Committee RANZCOG to try and estimate the failure rate of Filshie clip sterilisations carried out by gynaecologists in the state of Victoria, Australia.

\section{Materials and methods}

The total practising membership of the RANZCOG in the state of Victoria (277 Fellows) was surveyed. Each Fellow was asked whether he or she has personally had a failure (including procedures carried out by trainees under their supervision) or delivered or terminated a pregnancy which was associated with a Filshie clip sterilisation during the last 5 years. Sub-specialists in ultrasound were specifically asked whether they had scanned any pregnancies associated with Filshie clip sterilisation. All Fellows were asked if they would be prepared to be contacted by telephone to discuss any of these cases. A second personalised questionnaire and subsequently, if necessary, a third personalised letter was sent to non-responders. Any practitioner who did not respond to these three questionnaires received a personal telephone call from one of the two authors. A response was obtained from 276/277 practising Fellows (a response rate of $99.6 \%$ ). All pregnancies were identified and recorded. Each pregnancy was cross-checked to ensure that although reported by both the practitioner performing the sterilisation and the practitioner terminating or confining the woman, that it was only counted once.

The distributors of Filshie clips (Endovasive, Sydney, Australia) were contacted and they provided information on the number of Filshie clips sold in the state of Victoria between 1994 and 1998. They advised us that 30000 pairs of clips were sold during this period.

\section{Results}

Of the 276 Fellows who replied to the questionnaire, 223 $(80.8 \%)$ had no personal experience of failure, either as the person performing the sterilisation procedure or as an obstetrician. (Figure 1). Of the remaining 53 respondents, a total of 73 failures were identified resulting in a failure rate of 73 per 30000 sterilisation operations or $0.24 \%$. This can also be expressed as between 2 and 3 failures per 1000 sterilisations performed.

Of these failures, 29 on subsequent visualisation were confirmed as a failure of a 'properly applied' Filshie clip, and a further 14 cases were thought to be due to operator 
Figure 1 Data from a survey of the total practising membership of the Royal Australian and New Zealand College of Obstetrics and Gynaecologists (RANZCOG) in the state of Victoria

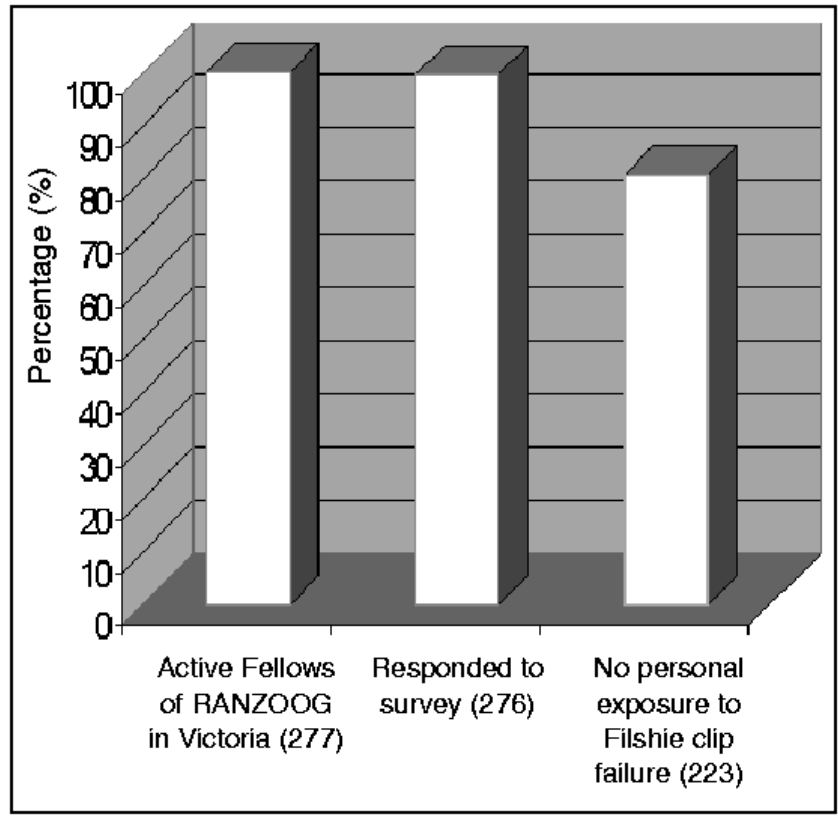

failure (misapplication of clip). In 30 instances, the reason for failure was not known, i.e. the tubes were not subsequently visualised. No ectopic pregnancies were reported.

\section{Discussion}

The limitation of the present study is that it depended on the recall of the gynaecologists surveyed. However, failure of a sterilisation is such a significant event that gynaecologists would remember this occurring to one of their patients, especially as such an event may occur only once or twice in any doctor's career. By approaching failure from both the operator's and the obstetrician's points of view, we believe that it is unlikely that we missed a significant number of failures. Even if we did not record all the failures, the magnitude of the numerator would not change significantly.

Whilst 30000 pairs of Filshie clips were sold during the study period, it is likely that the denominator is somewhat less than 30 000. Some clips may have been wasted, more than two clips may have sometimes been applied, whereas some clips may not have been used up at the end of the 5year period. However, even if 6000 clips were wasted, the failure rate would not be more than 3 per 1000 sterilisation operations $(73 / 24000=0.3 \%)$.

The quoted failure rates of 3 per 1000 sterilisations $^{2}$ are supported by the findings of the present study. This report confirms that failures seem to be sporadic within the state of Victoria, although one operator reported three failures.

The authors propose that as long as operating staff ensure regular calibration of the equipment, and operators are meticulously careful about placement of the clips, that Filshie clip sterilisation is an effective method of female sterilisation.

It is also recommended that a photograph illustrating correct application, the batch number of the clip(s) applied, and the number of the applicator used be recorded in the patient's medical records.

\section{Statements on funding and competing interests}

Funding. None declared.

Competing interests. G Kovacs was sponsored by Endovasive (Australian distributor) to present the findings of the present study at the Australian Gynaecological Endoscopy Society in 2000.

References

PH, Frances M, Paterson PJ. A comprehensive review of female sterilisation - tub occlusion methods. Clin Reprod Fertil 1985; 3: 81-97.

Tak Fore of the Australian Fedions. AFFPA Medical Task Force Guidelines. Med J Aust 1985; 143: 162-163.

Peterson HB, Xia Z, Hughes JM, et al. The risk of pregnancy after tubal sterilisation: finding for the US collaborative review of sterilisation. Am J Obstet Gynecol 1996; 174: 1161-1170. De Villiers VP. Post partum sterilisation with the Filshie titanium silicone-rubber clip an subsequent pregnancy. S Afr Med J 1987; 71: 498-499.

Birdsall MA, Wilson P, Pattison, NS. Female sterilisation: National Women's Hospital. N Z Med $J$ 1994; 107: 473-475.

Kjer JJ, Berild D. Laparoscopic sterilisation with Filshie clips. ACTA Obstet Gynecol Scand 1987; 66: 701-702

Filshie GM. Personal communication, 2 November 1998.

Sokal D, Gates D, Amatya R, Dominik R. Two randomized controlled trials comparing the Tubal Ring and Filshie Clip for tubal sterilization. Fertil Steril 2000; 74: 525-523. 